\title{
Electrospinning of Fibrous PHBV Tissue Engineering Scaffolds: Fiber Diameter Control, Fiber Alignment and Mechanical Properties
}

\author{
Ho-Wang Tong, Min Wang
}

\begin{abstract}
Poly(hydroxybutrate-co-hydroxyvalerate) (PHBV) fibrous membranes were fabricated through electrospinning. The effects of polymer solution properties including solution concentration and salt addition were investigated such that uniform fibers with diameters ranging from several hundred nanometers to a few micrometers were successfully produced. It was found that the fiber diameter increased with polymer solution concentration and drastically decreased with the addition of benzyl triethylammonium chloride. For each type of PHBV polymer solution, fibrous membranes with different fiber orientations such as random fibers, aligned fibers and double-layered structures consisting of aligned fibers were produced and the effect of solution properties on fiber alignment was analyzed. The mechanical properties of fibrous membranes with different fiber diameters and different fiber orientations were systematically investigated using tensile tests. The aligned fibers exhibited high tensile strength along the fiber direction.
\end{abstract}

\section{INTRODUCTION}

$\mathrm{E}$ LECTROSPINNING, a technology that can produce

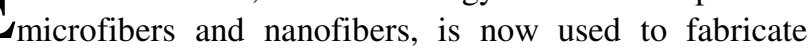
various types of fibrous scaffolds for tissue engineering (TE) applications [1-6]. Depending on the specific TE applications, ultrafine fibers of different diameters may be required and made. Since most biological tissues consist of nano-scaled fibers or fibrils, nanofibers are usually preferred to construct TE scaffolds in order to mimic the extracellular matrix (ECM) of various biological tissues [7]. For bone TE applications, nanoparticles of some calcium phosphates such as hydroxyapatite (HA) can be incorporated into electrospun fibers [8-10] because of the osteoconductive property of HA [11]. In such situations, microfibers were mostly produced because it was easier for microfibers to encapsulate nanoparticles than nanofibers to do so. The diameter of electrospun fibers varies and its effects on cell behavior have been investigated by different research groups [12-13]. The fiber alignment is another important factor that can influence cell response and hence determine the performance of fibrous scaffolds in some tissue engineering applications. Therefore, the effects of fiber alignment on cell behavior have also received considerable attention recently [14-15].

Manuscript received Mar 15, 2008. The work reported in this paper was supported by a research grant from The University of Hong Kong.

Ho-Wang Tong is with the Department of Mechanical Engineering, The University of Hong Kong, Pokfulam Road, Hong Kong (e-mail: h0227867@hkusua.hku.hk).

Min Wang is with the Department of Mechanical Engineering, The University of Hong Kong, Pokfulam Road, Hong Kong (corresponding author, phone: 8522859 7903; e-mail: memwang@ @ku.hk).
Apart from fiber size and orientation and their effects, mechanical properties of the electrospun fibers have often been investigated [16-17] because sufficient mechanical strength of the scaffolds is required. The fiber diameter, fiber alignment and mechanical properties of the scaffold are all important factors for a desirable TE scaffold. In our previous studies, techniques and facilities were developed for fabricating nonwoven $[8,9]$ and aligned-fiber TE scaffolds [10]. In the current study, we successfully electrospun fibers with diameters ranging from a few hundreds nanometers to a few micrometers and with different fiber orientations. The mechanical properties scaffolds of the fibers with different diameters and orientations were evaluated systematically.

\section{MATERIALS AND METHODS}

\section{A. Materials}

Poly(hydroxybutrate-co-hydroxyvalerate) (PHBV) with a molecular weight of 310,000 and containing $2.9 \mathrm{~mol} \%$ of 3-hydroxyvalerate was from Tianan Biologic Material Ltd., China. Benzyl triethylammonium chloride (BTEAC) was commercially available (Fluka, Germany). The solvent, chloroform, was of analytical grade. The polymer (PHBV), organosoluble salt (BTEAC) and solvent were used for eletrospinning in the as-received state.

\section{B. Solution preparation}

To prepare solutions for electrospinning, PHBV was dissolved in chloroform at $50^{\circ} \mathrm{C}$ with gentle stirring for 90 minutes using a hotplate magnetic stirrer. Three groups of solutions were prepared: (1) $2 \mathrm{~g}$ of PHBV in $10 \mathrm{ml}$ of chloroform, (2) $1.5 \mathrm{~g}$ of PHBV in $10 \mathrm{ml}$ of chloroform, and (3) $1.5 \mathrm{~g}$ of PHBV in $10 \mathrm{ml}$ of chloroform with an addition of 0.03 $\mathrm{g}$ of BTEAC. The first, second and third groups of solutions were designated as solution $\mathrm{A}, \mathrm{B}$ and $\mathrm{C}$, respectively.

\section{Electrospinning}

Each group of solutions was used for electrospinning to produce three types of fibrous structures, namely, randomly oriented fibrous membrane, single-layer aligned fibrous membrane, and double-layered fibrous membrane consisting of aligned fibers. Details on fabricating random fibers and aligned fibers can be found in our previous publications [810]. The electrospinning processing conditions were: solution feeding rate $=3 \mathrm{ml} / \mathrm{h}$; voltage $=15 \mathrm{kV}$; needle inner diameter $=0.5 \mathrm{~mm}$; distance between the needle tip and collector $=10$ $\mathrm{cm}$; rotational speed of the cylinder $=4167 \mathrm{rpm}$. 


\section{Morphological characterization}

Electrospun fibers were characterized using a scanning electron microscope (SEM, Stereoscan 440, UK) and each SEM micrograph was analysed using an image tool (UTHSCSA Image Tool). To characterize the fiber size, fifty individual fibers were selected from the SEM micrograph and their diameters were measured using the image tool. To characterize the degree of fiber alignment, fifty individual fibers were selected and the angles between the fibers and the longitudinal axis of the fiber collecting cylinder were determined using the image tool. The angle of $90^{\circ}$ indicated perfect fiber alignment on the fiber collecting cylinder.

\section{E. Mechanical characterization}

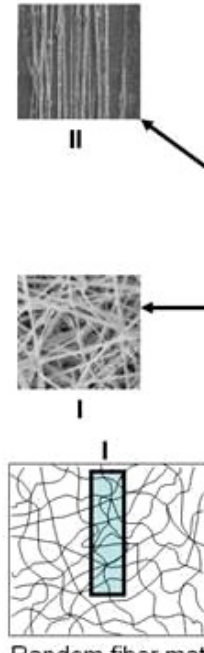

Random fiber mat

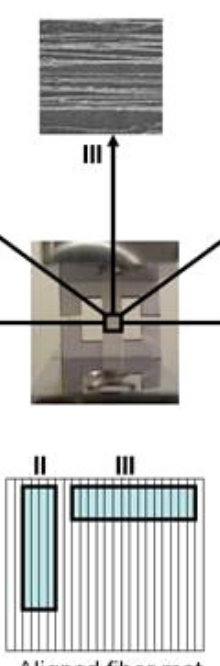

Aligned fiber mat

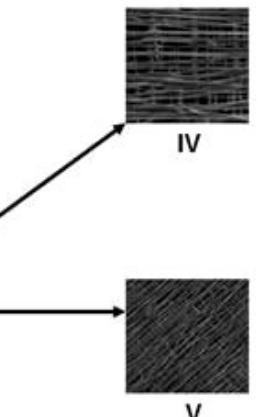

V

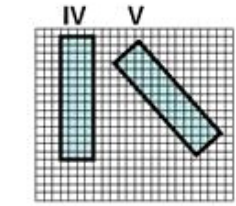

Double-layered fiber mat
Fig. 1 Specimens with different types of fiber orientation

Mechanical characterization was conducted by subjecting electrospun fibrous membranes to tensile testing. The specimens were divided into five groups, as shown in Fig.1: (I) Randomly oriented fibrous membranes, (II) Aligned fibers tested in the longitudinal direction (i.e. in the direction of cylinder rotation), (III) Aligned fibers tested in the perpendicular direction (i.e. at $90^{\circ}$ to the direction of the cylinder rotation), (IV) Double-layered structures consisting of a horizontally aligned fiber layer and a vertically aligned fiber layer tested in either the parallel or the perpendicular direction, and (V) Double-layered structures tested at $45^{\circ}$ to the direction of the cylinder rotation. All test specimens were in the form of rectangular shape $(5 \times 30 \mathrm{~mm}$ in dimension $)$ cut from fibrous membranes. Fifteen specimens were tested for each orientation group (I to V), with five specimens from each solution group (A to $\mathrm{C}$ ). The thickness of each fibrous membrane was measured using a non-contact method. The tensile testing was performed using an Instron tester (Model 5848) with a load cell of 10 N. A cross-head speed of 2 $\mathrm{mm} / \mathrm{min}$ was used for all tests. Each specimen was tested to the point of failure and the ultimate tensile strength, Young's modulus and elongation at break were obtained.

\section{RESULTS AND DISCUSSION}

\section{A. Effects of solution properties on fiber diameter and fiber alignment}
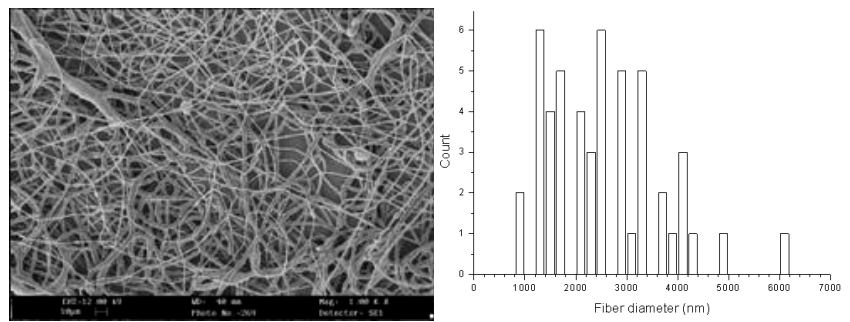

(a) fibers electrospun from solution $\mathrm{A}$
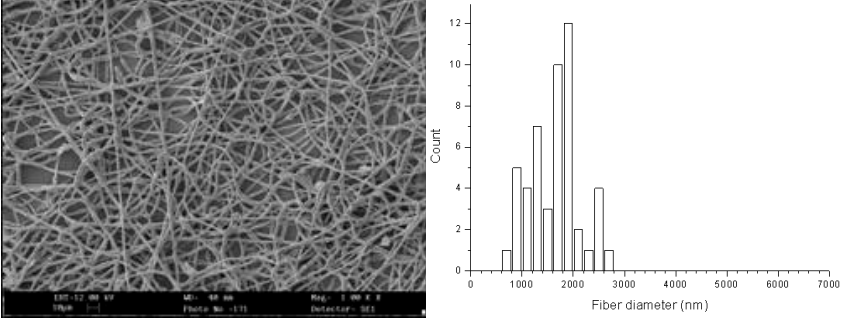

(b) fibers electrospun from solution $\mathrm{B}$
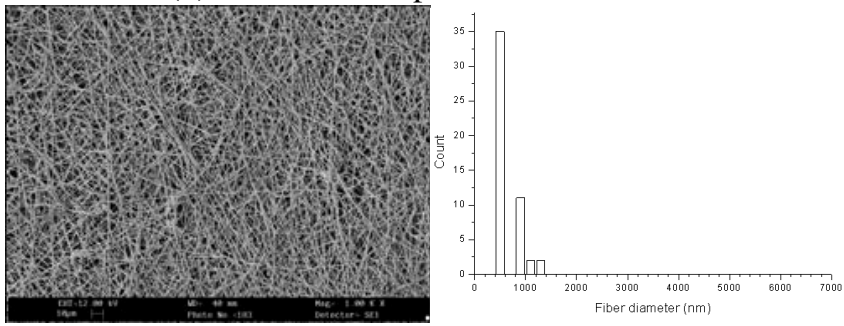

(c) fibers electrospun from solution $\mathrm{C}$

Fig. 2 Morphology of random fibers (left) and the fiber diameter distribution (right)

SEM micrographs and the corresponding fiber diameter distributions of randomly oriented fibers electrospun from solutions $\mathrm{A}, \mathrm{B}$ and $\mathrm{C}$ are shown in Fig.2. When the solution concentration of PHBV was increased from $15 \% \mathrm{w} / \mathrm{v}$ (solution B) to $20 \% \mathrm{w} / \mathrm{v}$ (solution $\mathrm{A}$ ), the average fiber diameter increased from $1662 \pm 490 \mathrm{~nm}$ to $2502 \pm 1123 \mathrm{~nm}$. However, when $0.03 \mathrm{~g}$ of BTEAC was added into the $15 \% \mathrm{w} / \mathrm{v}$ PHBV solution (solution $\mathrm{C}$ ), the average diameter of electrospun fibers was drastically decreased to $603 \pm 250 \mathrm{~nm}$. These results have clearly shown that decreasing solution concentration or adding the salt could effectively decrease the electrospun fiber diameter. With increased PHBV concentration, the polymer chain entanglements within the solution became greater. Therefore, it was more difficult for the charges on the polymer jet to stretch the jet into finer fibers. Addition of BTEAC increased the conductivity of the polymer jet, which facilitated the stretching of the jet by the electric force during electrospinning.

When the electrospun fibers were collected by the high speed rotating cylinder [10] instead of the flat conductive plate [9], the fibers could be quickly taken up by the cylinder and hence aligned fibers could be obtained. Fig. 3 shows the 
morphology of aligned fibers and the degree of fiber alignment. All fibers electrospun from solution A were aligned between $80^{\circ}$ and $100^{\circ}$ relative to the longitudinal axis of the rotating cylinder. Although the degree of alignment of the fibers electrospun from solution B was not as satisfactory as that of the fibers electrospun from solution $\mathrm{A}$, there were still nearly half of the fibers aligned between $80^{\circ}$ and $100^{\circ}$ relative to the longitudinal axis of the rotating cylinder. However, the fibers electrospun from solution $\mathrm{C}$ did not show a fiber alignment, even though they were collected by the same rotating cylinder at the same rotating speed. It is clear that the degree of fiber alignment was improved by increasing PHBV concentration but worsened by the addition of BTEAC. This phenomenon will be explained in our future publication due to space limitation for this paper.
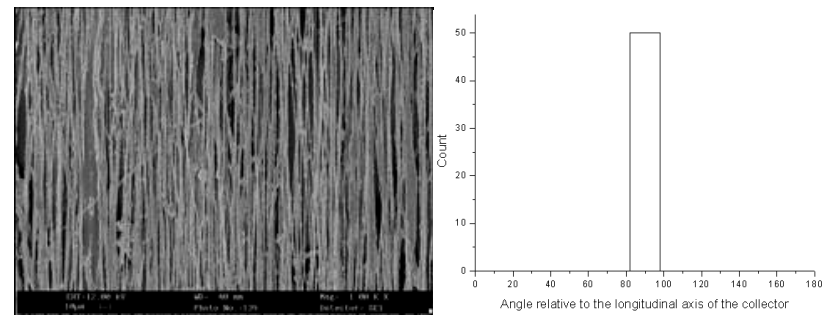

(a) fibers electrospun from solution $\mathrm{A}$
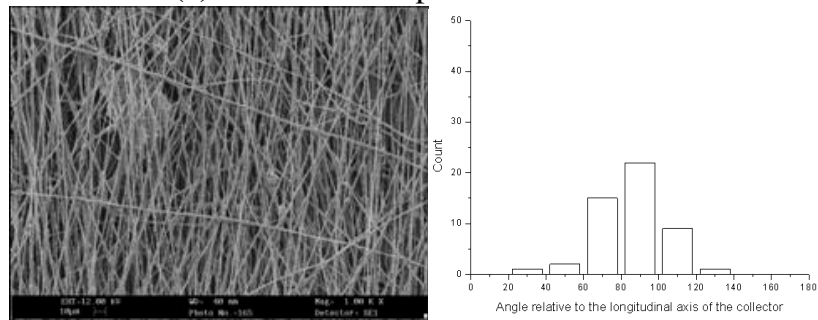

(b) fibers electrospun from solution B
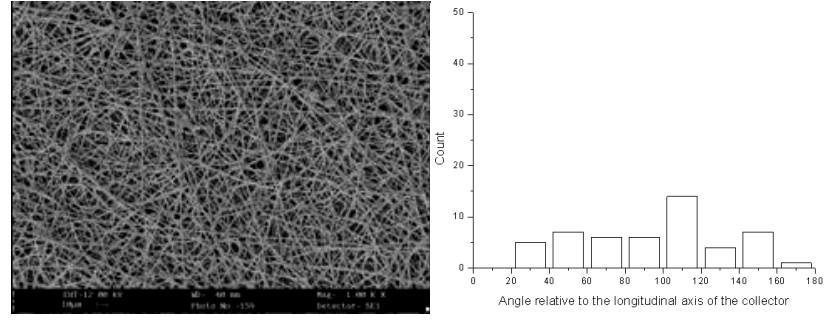

(c) fibers electrospun from solution $\mathrm{C}$

Fig. 3 Morphology of aligned fibers (left) and the degree of fiber alignment (right)

\section{B. Mechanical properties of fibrous membranes with different fiber sizes and fiber orientations}

Fig.4 shows tensile properties of electrospun fibrous membranes with different fiber diameters (from solutions A to C) and of different orientations (types I to V). The average ultimate tensile strength (Fig. 4a), Young's modulus (Fig. 4b) and elongation at fracture (Fig. 4c) were determined for each group.

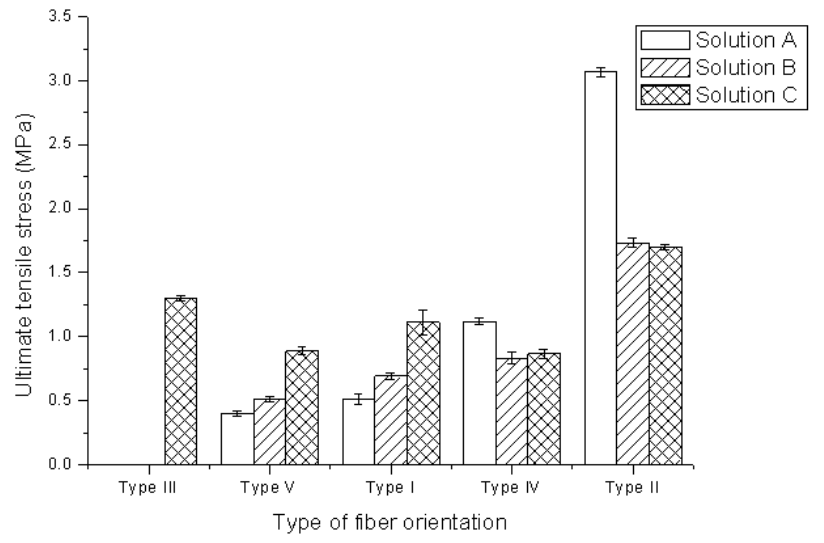

(a) ultimate tensile strength

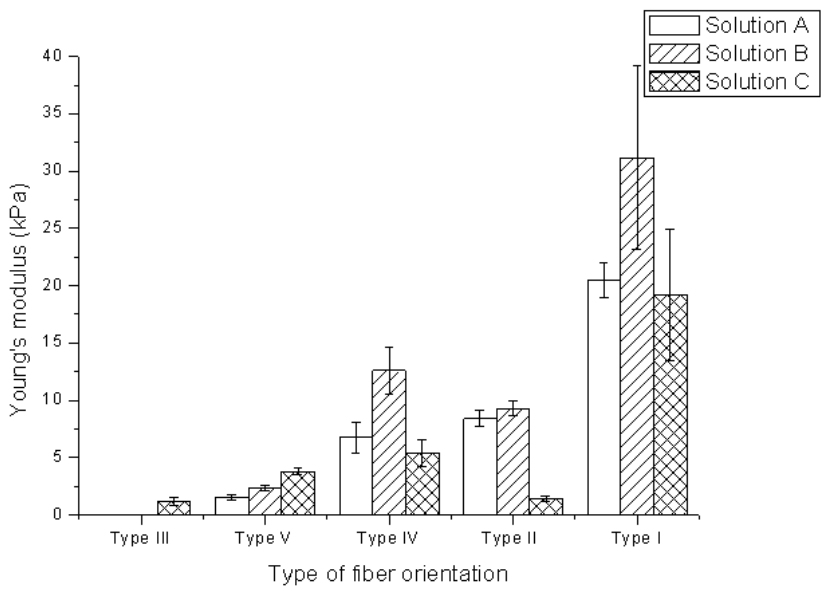

(b) Young's modulus

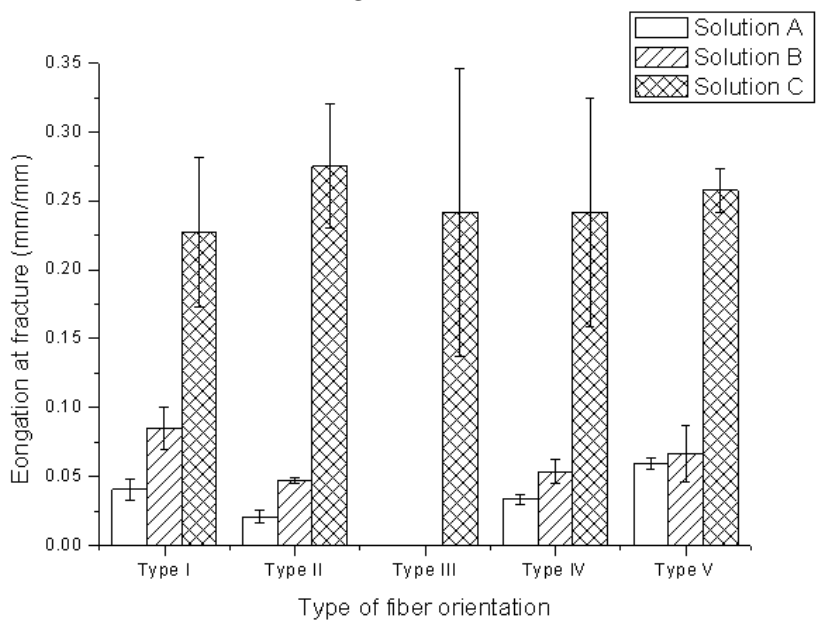

(c) Elongation at fracture

Fig. 4 Tensile properties of electrospun fibrous membranes

When the fibers were electrospun from solution A, the type II specimens exhibited the highest ultimate tensile strength, followed by the type IV, I, V and III specimens. Fibrous membranes electrospun from solution B also showed a similar trend, although the trend was not very obvious. This trend was not applicable for fibers electrospun from solution C, probably due to the low alignment effect. Seemingly in this study, the randomly oriented fibrous membranes (i.e. type I) 
exhibited the highest Young's modulus among different fiber orientations, no matter what polymer solution (A, B or C) was used. The fiber orientation did not play a significant role in affecting the elongation at break, but the fiber diameter did. The fibers electrospun from solution $\mathrm{C}$, which represented the smallest fiber dimension, exhibited the highest elongation at break. Therefore, fibers with smaller diameters would be desirable if one needs to produce a tough fibrous membrane. The successful control of fiber diameter and alignment should render fibrous scaffolds with different mechanical properties. These results are very useful fabricating TE scaffolds which mimic the mechanical properties of different tissues. For example, due to the high tensile strength, the highly aligned fibrous scaffold should be useful for regenerating skin, tendon or ligaments because these tissues are frequently subjected to tensile forces.

\section{CONCLUSIONS}

PHBV fibers with fiber diameters ranging from several hundred nanometers to a few micrometers could be electrospun by varying the polymer solution properties. A high speed rotating drum could be used to fabricate membranes consisting of aligned fibers through electrospinning, but the degree of fiber alignment could be affected by solution properties. Mechanical properties of electrospun fibrous membranes could be assessed using tensile tests. The mechanical properties were affected by the fiber diameter and fiber alignment.

\section{ACKNOWLEDGEMENT}

This work was supported by The University of Hong Kong (HKU). Ho-Wang Tong thanks HKU for providing him with a research studentship. Assistance provided by technical staff in the Department of Mechanical Engineering, HKU, is acknowledged.

\section{REFERENCES}

[1] N. Ashammakhi, A. Ndreu, A. M. Piras, L. Nikkola, T. Sindelar and H. Ylikauppila, "Biodegradable nanomats produced by electrospinning: expanding multifunctionality and potential for tissue engineering", $J$ Nanosci Nanotechno, Vol.7 (2007), pp862-882.

[2] U. Boudriot, R. Dersch, A. Greiner and J. H. Wendorff, "Electrospinning approaches toward scaffold engineering - brief overview", Artif Organs, Vol.30 (2006), pp785-792.

[3] C. Burger and B. Chu, "Functional nanofibrous scaffolds for bone reconstruction", Colloid Surface B, Vol.56 (2007), pp134-141.

[4] S. Y. Chew, Y. Wen, Y. Dzenis and K. W. Leong, "The role of electrospinning in the emerging field of nanomedicine", Curr Pharm Design, Vol.12 (2006), pp4751-4770.

[5] E. J. Chong, T. T. Phan, I. J. Lim, Y. Z. Zhang, B. H. Bay and S. Ramakrishna, "Evaluation of electrospun PCL/gelatin nanofibrous scaffold for wound healing and layered dermal reconstitution", Acta Biomater, Vol.3 (2007), pp.321-330.

[6] J. Venugopal, S. Low, A. T. Choon and S. Ramakrishna, "Interaction of cells and nanofiber scaffolds in tissue engineering", J Biomed Mater Res B, Vol.84 (2008), pp.34-48.

[7] C. M. Vaz, S. van Tuijl, C. V. C. Bouten and F. P. T. Baaijens, "Design of scaffolds for blood vessel tissue engineering using a multi-layering electrospinning technique", Acta Biomater, Vol.1 (2005), pp.575-582.
[8] H. W. Tong and M. Wang, "PHBV-based composite nanofibers for bone tissue engineering", in Proceedings of the $10^{\text {th }}$ Annual Conference on Ceramics, Cells and Tissues, Faenza, Italy, 2006, pp192-200.

[9] H. W. Tong and M. Wang, "Effects of processing parameters on the morphology and size of electrospun PHBV micro- and nano-fibers", Key Eng Mat, Vol.334-335 (2007), pp.1233-1236.

[10] H. W. Tong and M. Wang, "Electrospinning of aligned biodegradable polymer fibers and composite fibers for tissue engineering applications", Journal of Nanoscience and Nanotechnology, Vol.7 (2007), pp.3834-3840.

[11] M. Wang, "Developing bioactive composite materials for tissue replacement", Biomaterials, Vol.24 (2003), pp.2133-2151.

[12] A. S. Badami, M. R. Kreke, M. S. Thompson, J. S. Riffle and A. S. Goldstein, "Effect of fiber diameter on spreading, proliferation, and differentiation of osteoblastic cells on electrospun poly(lactic acid) substrates", Biomaterials, Vol.27 (2006), pp.596-606.

[13] M. Chen, P. K. Patra, S. B. Warner and S. Bhowmick, "Role of fiber diameter in adhesion and proliferation of NIH $3 \mathrm{~T} 3$ fibroblast on electrospun polycaprolactone scaffolds", Tissue Eng, Vol.13 (2007), pp.579-587.

[14] S. Y. Chew, R. Mi, A. Hoke and K. W. Leong, "The effect of the alignment of electrospun fibrous scaffolds on Schwann cell maturation", Biomaterials, Vol.29 (2008), pp.653-661.

[15] C. A. Mills, J. G. Fernandez, E. Martinez, M. Funes, E. Engel and A. Errachid, "Directional alignment of MG63 cells on polymer surfaces containing point microstructures", Small, Vol.3 (2007), pp.871-879.

[16] M. L. Cheng, C. C. Lin, H. L. Su, P. Y. Chen, and Y. M. Sun, "Processing and characterization of electrospun poly(3-hydroxybutyrate-co-3-hydroxyhexanoate) nanofibrous membranes", Polymer, Vol.49 (2008), pp.546-553.

[17] S. J. Lee, S. H. Oh, J. Liu, S. Soker, A. Atala and J. J. Yoo, "The use of thermal treatments to enhance the mechanical properties of electrospun poly(E-caprolactone) scaffolds", Biomaterials, Vol.29 (2008), pp.1422-1430. 